\title{
没食子酸衍生物对人血小板膜 流动性影响的苂光探剂研究
}

\author{
祁领平 张家俊 吴荣青
}

（北京中医学院中心实验室）

没食子酸是“活血化湶”常用中药赤芺中的一种成分. 以前的实验资料指出：赤芳具有明 显的抗血小板聚集、促进血栓溶解等作用,也指出没食子酸及其衍生物没食子酸丙酯和没食子 酸异丁酯具有类似作用 ${ }^{[1]}$. 在诱发血小板聚集反应的过程中，伴有血小板瞙脂质的物理状态 发生变化，一些药物的抗血小板聚集是通过调整血小板膜的物理状态，特别是脂质一脂质和/ 或脂质一蛋白之间的相互作用 ${ }^{[2-9]}$. 本文是应用荧光探剂观察赤芳成分没食子酸衍生物（没食 子酸丙酯和没食子酸异丁酯) 对血小板膜流动性的影响, 以了解药物抗血小板聚集作用的机 理.

\section{一、材料与方法}

村料 ADP 及苂光探剂 ANS (1-苯胺-8-菜磺酸) 和 $\mathrm{DPH}(1,6-$ 二苯酚-1, 3, 5-已 三烯）为 Sigma Chemical Co 产品, 苂光探剂 NPN ( $\mathrm{N}$-苯基-1-苯胺) 为 Kodak 产品, Sepharose 2B 为瑞典 Pharmacia 产品. 其它试剂均为分析纯(北京化工厂产品).

没食子酸丙酯和没食子酸异丁酯是天津医药工业研究所赠送 ${ }^{[6]}$.

血小板分离方法按文献稍加改进,血小板计数约 $1.0 \times 10^{9}-1.1 \times 10^{9}$ 细胞 $/ \mathrm{ml}$.

血小板标记实验中采用 ANS, NPN 和 DPH 三种茨光探剂, ANS, NPN 标记方 法见参考文献 [7], 并加以改进, DPH 标记见参考文献 [2]. ANS 和 NPN 是研究膜相变 的较理想的指示剂, ANS 结合于膜和水相的交界面, 为探测脂质双层甘油区的茨光探剂.NPN 结合于脂质双层的碳氢链区. DPH 为测量苂光偏振探剂, 它结合于深部碳氢链区.

苂光强度和苂光偏振测量 使用日立 MPF-4 型苂光分光光度 计 及 偏振 附 件, 用 LAUDA-C 3 (西德) 循环恒温水浴装置保温和自行改装的 CIT-2 型数字温度计监测比色杯温 度, 精度为 $0.5^{\circ} \mathrm{C}$. 以 ANS 为苂光探剂时, 激发波长和发射波长分别为 $398 \mathrm{~nm}$ 和 $478 \mathrm{~nm}$. 以 NPN 为荧光探剂时, 为 $349 \mathrm{~nm}$ 和 $426 \mathrm{~nm}$. 以 $\mathrm{DPH}$ 为荧光探剂时, 为 $364 \mathrm{~nm}$ 和 432 nm.

膜脂相变温度的测定方法见参考文献 [7]. 膜脂的相变是指构成生物膜的脂质双层分子 凝胶相(有序排列)和液晶相 (无序排列)之间的转变, 低于相变温度范围时星凝胶相, 高于相变 温度范围时呈液晶相, 在相变温度范围内, 膜脂中凝胶相与液晶相共存. 相变温度降低, 反映 膜的流动性增加, 反之, 相变温度增高, 则反映膜的刚性增加.

荧光偏振 $\boldsymbol{p}$ 或荻光各向异性 $\boldsymbol{r}$ 的测量用下述公式:

本文1986 年10月6日收到。 


$$
p=\frac{I v v-G I v h}{I v v+G I v h}, \quad r=\frac{I v v-G I v h}{I v v+2 G I v h},
$$

Ivo 和 $I v h$ 是当起偏振器在垂直方位时, 分别将检偏振器放在垂直和水平方位测得的茨光强 度. $G=I h v / l h h$ 是校正因子. 本文使用荻光各向异性来描述脂质双层的微环境变化, $r$ 的 数值增大, 则表示环境的微粘度增大, 膜脂双层的流动性降低.

\section{二、结 果}

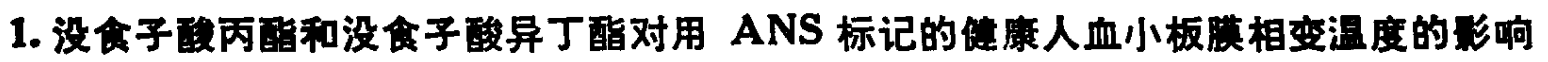

图 1 表示以 ANS 为探剂时苂光强度与温度变化的关系. 当没食子酸丙酯终浓度 为 1.67 $\mathrm{mmol} / \mathrm{l}$ 时, 血小板膜的相变温度降低约 $4.5^{\circ} \mathrm{C}$, 而没食子酸异丁酯降低约 $7^{\circ} \mathrm{C}$.

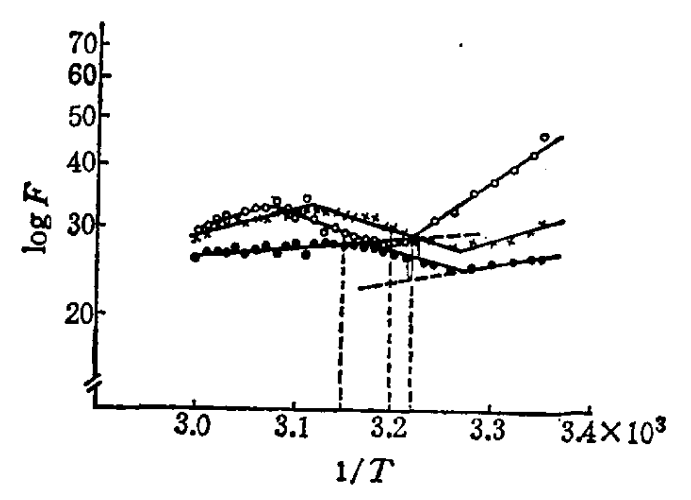

图 1 以 ANS 为探剂时没食子酸丙酯和没食 子酸开丁酯对人血小板膜相变温度的影响 ○一一 - 0 对琞; $\times$ - $\times$ 没食子酸丙酯; 没食子酸异丁醌

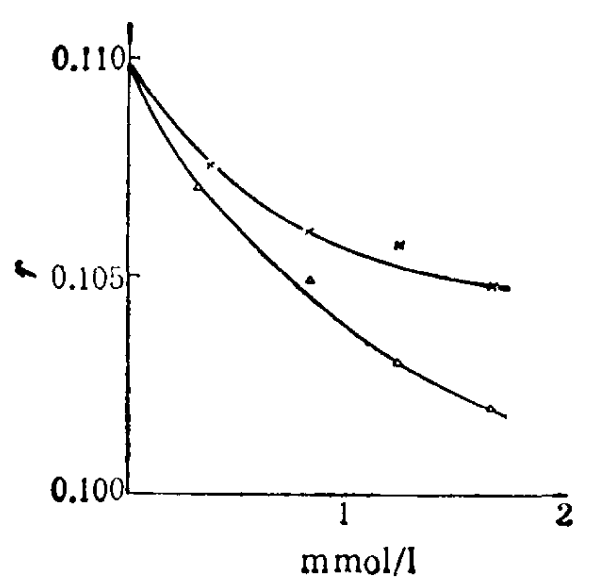

图 3 药物浓度对以 DPH 为荧光探剂的人 血小板膜的荧光各向异性的影响

血小板悪液浓度为 $0.73 \times 10^{9}$ 细胞 $/ \mathrm{ml}$.

$x$ 一 $\times$ 没食子酸丙酷; $\Delta-\Delta$ 没食子酸异丁酯

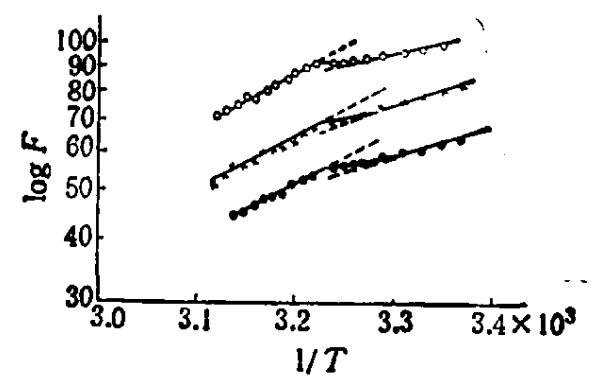

图 2 以 NPN 为探剂时没食子酸丙酯和没食 子酸开丁酯对人血小板膜相变温度的影响

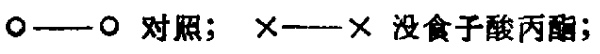
没食子酸异丁醌

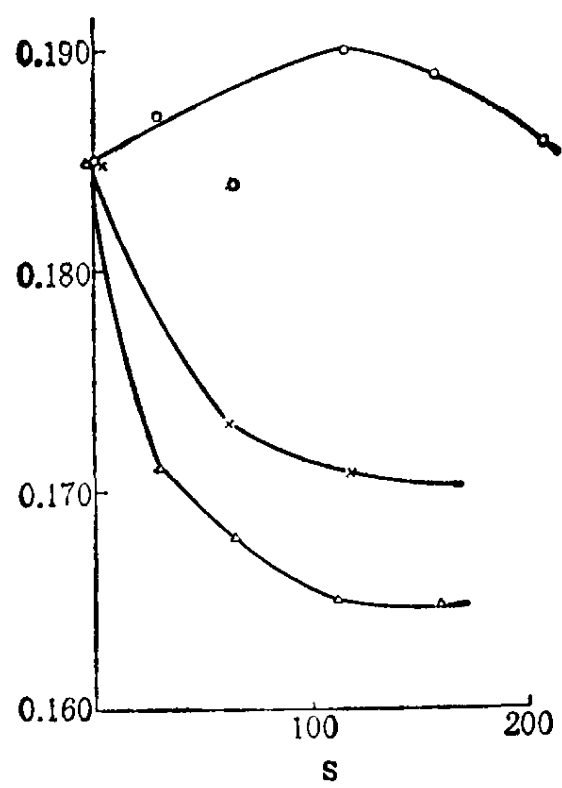

图 4 药物对 ADP 诱导 DPH 标记的血小板聚集 时荧光各向异性变化的影响

$0-0 \quad 0.5 \mu \mathrm{mol} / \mathrm{l} \mathrm{ADP} ; \times \longrightarrow \times \mathrm{ADP}+1 \mathrm{mmol} / 1$ 没 食子酸丙醌; $\Delta-\Delta \mathrm{ADP}+1 \mathrm{mmol} / 1$ 没食子酸异丁酯 


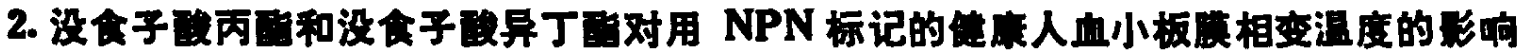

图 2 表示当药物终浓度为 $1.67 \mathrm{mmol} / 1$ 时, 没食子酸丙酯使血小板膜相变温度降低约 $3.6^{\circ} \mathrm{C}$, 没.食子酸异丁酯降低约 $5^{\circ} \mathrm{C}$.

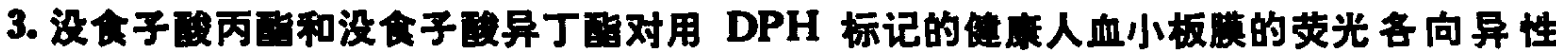
（r）的到响结果如图 3 所示当药物浓度增加时 (终浓度在 $0-1.67 \mathrm{mmol} / 1$ 范围内), 两种 药物均可使健康人血小板膜的苂光各向异性降低,即使膜的流动性增加, 没食子酸异丁酯的作 用比没食子酸丙酯更为明显.

\section{4. 没食子酸两西和没食子酸异丁西对用 ADP 婇导血小板聚集时膜流动性的影响} 结果如图 4, 在血小板悬液中加人 ADP（诱导血小板聚集因素之一)后,血小板膜的苂光各向 异性增加,即膜的流动性降低. 加人药物后, 则 ADP 的诱导聚集作用受到抑制,血小板膜流 动性增加. 没食子酸异丁酯的作用较没食子酸丙酯明显.

单纯加人 ADP 后, DPH 和 ANS 标记的血小板膜荧光强度均无明显变化.

\section{三、讨 论}

本文实验结果证明,没食子酸丙酯和没食子酸异丁酯都具有增加血小板膜流动性的作用, 联系前文的工作,本实验也符合量效关系与构效关系规律,从多种没食子酸衍生物的上述作用 可推测衍生物母核取代碳链不同时,含碳数愈高，亲脂性愈强时，增加膜流动性也愈明显. 这 种现象可能有利于改善血小板的生理功能. 本文实验在保持血小板活性的情况下，采用茨光 探剂标记的完整血小板, 探讨该膜的流动性变化及上述药物的作用机理.

前人曾研究凝血酶、ADP 及纤维蛋白元等因素诱导血小板聚集的过程中膜的微环 境变 化 ${ }^{[2-4]}$. 认为 DPH 标记的活性血小板荧光各向异性 (或资光偏振) 的增加, 反映了血小板形 状变化与膜的流动性变化的关系. 膜脂质的敃粘度的增加, 反映了膜脂质层内脂质一脂质相互 作用和脂质排列密度的增加，即膜脂刚性增加．证实膜脂刚性的增加是引起血小板聚集的主 要因素之一. 分析其原因, 首先是脂质可能调节膜受体的活性，由于脂质-脂质间相互作用的 增加,使膜蛋白(包括受体蛋白)更多地暴露于膜的水相表面环境. 其次,膜磷脂可能更容易与 内源磷脂酶 $\mathrm{A} 2$ 接触,该酶活性随着脂质微粘度的增加明显激活.

没食子酸丙酯和没食子酸异丁酯具有增加健康人血小板膜流动性的作用. 该现象说明药 物抗血小板聚集和促进血栓溶解的作用,可能是通过调整早期膜脂质物理状态的变化,增加了 膜的流动性，抑制膜脂刚性的增加，而导致抑制血小板聚集作用.

同时,以上实验也可帮助了解药物分子结构与血小板功能的关系, 从分子药理学方面提供 阐述中药作用机理的途径, 这对提高疗效、降低毒性、改造药物分子结构提供了有益的根据。

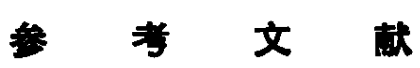

[1] 王继等, 中西医结合杂志, 4(1984), 12: 745.

[ 2 ] Nathan, I. et al., J. Biol. chem., 254(1979), 9822-9828,

[ 3] Nathan, 1. et al., Biochim. Biophys. Acta, 598(1980), 417-421.

[4] Kowalska, M. A. et al., Biochim. Biophys. Acre, 729 (1983), 275-280.

[5] Steiner, M., Biochim. Biophys. Acta, 640(1981), 100-105.

[6] Tangen, O. et al., Scand. J. Haemat, 10(1973), 96-105.

[7] 祁颂平等。生物化学与生物物理学报, 18(1986),246-251. 